\title{
Priority Data Transmission Schemes for a Wireless-9069 Sensor Network on BAN
}

\author{
Sergio Martínez ${ }^{1}$, Mario Rivero ${ }^{2}$, Laura Garay ${ }^{1}$, Issis Romero ${ }^{1}$ \\ ${ }^{1}$ Instituto Politécnico Nacional, \\ Unidad Profesional Interdisciplinaria en Ingeniería y Tecnologías Avanzadas, \\ CDMX, Mexico \\ ${ }^{2}$ Instituto Politécnico Nacional, Centro de Investigación en Computación, \\ CDMX, Mexico \\ smartinezc1403@alumno.ipn.mx, lgaray@ipn.mx, \\ iromero@ipn.mx, mriveroa@ipn.mx
}

\begin{abstract}
Among the most recurrent Wireless Sensor Network (WSN) employment is the biopotential signals monitoring (e.g. electrocardiograms, electroencephalograms, electrogastrograms) that allows transmitting the body organ response from a person. Within the WSN's focused on bioelectrical studies, the energy analysis is essential indeed, given that sensors are devices of minimal dimensions and implies batteries tend to be small and have short-lifetime. In the present research we proposed two priority schemes to enhance the network performance by giving transmission priority to certain nodes. In a first scheme, nodes have different priorities according to the data type they are reporting on. Hence, nodes that sense more relevant data have higher priority to use the channel compared to nodes reporting less important data. For the second scheme, the aim is to prolong the system lifetime by assigning higher transmission probability to nodes with higher residual energy levels while nodes with little energy left, perform fewer transmissions. We show that the performance of the system is indeed enhanced by the introduction of such schemes.
\end{abstract}

Keywords: Wireless Sensor Network (WSN), Body Area Network (BAN), Cognitive Ratio (CR).

\section{Introduction}

Nowadays, the implementation of wireless and ambulatory technologies in medical applications has important relevance [1]. Take into consideration, the multiple areas joint effort to work on e-health applications, which primary goal is to increase life quality of people. Within e-health, it is required the gauge of constant signals emitted by the body. That is why tools are required that are capturing the electrical measures generated by the human being. These are known as bioelectrical studies. For biopotential signals monitoring, different solutions have emerged and an alternative relies on wireless sensor networks [2].

However, based on [3] and [4] the WSN confront an inherent conflict that intrinsically depends on batteries dimensions and capacities. In this context, batteries require an important energy performance analysis given that life-battery 
is shorter than current energy systems [5]. Nevertheless, studies of network energy

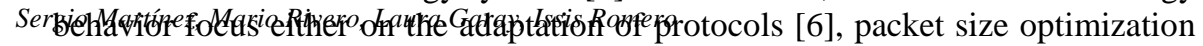
[7] or materials properties analysis giving greater capacity [8]. The present research proposed to design and simulate the energy performance of a WSN focused on the study of biopotential signals.

We analyze that in practical applications two common situations are presented, in one there are sensors that have priority over others because they are giving attention to a specific body area, consequently exist nodes that have more importance than others generating the priority based scheme. In other case, there are medical applications where the aim is to extend the lifetime batteries as much as possible. This second scheme requires an analysis of the remaining battery energy giving rise to the residual energy based scheme.

\section{System Design and Parameters Description}

A wireless sensor network is a set of devices placed in a certain area capable of performing basic operations, sensing and communicate with other sensors [9]. Let both describe a bioelectrical potential as the representation of the ion flow through the cell membrane and can be measured by invasive or non-invasive nodes for nerves and muscles in the human body [10].

The proposed system is composed of a set of nodes in sensors that measure bioelectrical signals assuming that all are placed in the body of a single person. The intercommunication distance responds to less than $3.28 \mathrm{ft}$, thus the sensors work on a body area network (BAN) [5] with e-health application. The system is designed considering that any biopotential study cited in the table bellow can be transmitted.

Table 1. Most used bioelectrical studies [11].

\begin{tabular}{llll}
\hline Study & Description & Voltage & Frequency $(\mathrm{Hz})$ \\
\hline ECG & $\begin{array}{l}\text { Electrocardiogram. Representation of heart electrical } \\
\text { signals }\end{array}$ & $0.5-4 \mathrm{mV}$ & $0.01-250 \mathrm{~Hz}$ \\
EEG & $\begin{array}{l}\text { Electroencephalogram. Depiction of electrical signals } \\
\text { produced by the brain. }\end{array}$ & $5-300 \mu \mathrm{V}$ & $150 \mathrm{~Hz} \mathrm{DC}$ \\
EGG & $\begin{array}{l}\text { Electrogastrogram. It is the representation of the } \\
\text { electrical signals produced by the stomach. }\end{array}$ & $10 \mu \mathrm{V}-1 \mathrm{mV}$ & $1 \mathrm{~Hz} \mathrm{DC}$ \\
EMG & $\begin{array}{l}\text { Electromyogram. Measures the electrical activity of } \\
\text { muscles. }\end{array}$ & $0.1-5 \mathrm{mV}$ & $10 \mathrm{KHz}$ \\
\hline
\end{tabular}

\subsection{Communication Parameters}

The following describes the general properties of the communication scheme used in the network:

- The communication scheme or protocol is TDMA since it requires a minimum hardware-software processing for its implementation and considers a deterministic system assuring the information transmission of each node in its respective slot.

- A hybrid network is understood in this project when it is used two different kinds of networks. The primary network consists of sensors that transmit continuously. On the other hand, the secondary network will transmit sporadically (i.e. event monitoring)
Research in Computing Science 138 (2017)

ISSN 1870-4069 
- The cognitive radio in the communication system network design is presented

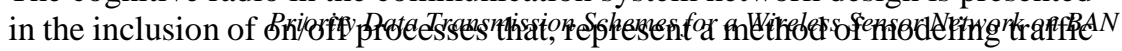
in stationary communications networks. To consider on/off processes, the following premises must be considered:

- The nodes of the continuous monitoring network (primary network) will be on for a specific period called time on.

- There will also be time periods for primary network nodes where they will be 'turned off'. In this sense, these periods are known as time off. When primary network has some turned off node, the respective slot will be free to transmit information that is generated from secondary network nodes. It is very important to indicate that the sensors work all the time and implies energy consumption at any time.

The communication protocol has as reference the work done in [12] and [13] where both focus on epilepsy, however at the present design is open to any biopotential study cited in Table 1.

\section{$3 \quad$ Energy Analysis}

There is clearly a great compromise between the information amount tolerance that can be lost from continuous monitoring and the energy consumption reduction. We estimate results for knowing in detail the network behavior and to carefully analyze this dependence. We consider $M$ as the number of slots for each frame.

To specify the quantitative analyzes, it is considered to evaluate the data in specific values. Let the primary network be an electroencephalogram composed of a set of 22 nodes. The secondary network will be monitoring a 4-channel electrogastrogram. Thus: $M=22$ nodes and $n_{e}=4$ event monitoring nodes (secondary network nodes).

\subsection{Energy Consumption And Packet Loss For WSN}

In the primary network there are in total $M$ nodes and the information will be transmitted continuously. In this case, it is remembered that it is a deterministic protocol so that each of the nodes of the electroencephalogram has a reserved slot to transmit. However, when the on/off processes are included, there is a change in the energy consumption derived from the on and off times. Therefore, the energy in the primary network is analyzed through two cases:

- Case 1. The node is active. The node is on (i.e. slot is related to an on time) and the usual energy transmission is taken. This energy will be evaluated by

$E_{[t]]}$ referring to the unit of energy consumption per packet transmission.

- Case 2. The node is in sleep mode. It refers to the node being switched off and therefore not transmitting EEG information. However, when the sensor is on (even if not send data), a certain amount of energy is being used which is much smaller than the active node. The energy is determined by:

$$
\left.E_{s}=0.1 E_{[t]}\right],
$$

where

$E_{s}=$ Sleep node energy consumption, 
$E_{[t x]}=$ Active node energy consumption

Sergio Martínez, Mario Rivero, Laura Garay, Issis Romero

The WSN performance is evaluated by variables $E_{[t x]}$ (for energy consumption) and Packets (for system loss). In both cases, there is a probability that the packet has been successfully transmitted. This is defined as the probability of successful packet transmission.

The secondary network is based on the $n_{e}$ nodes occupied. In numerical terms, for this analysis are four. In order to be able to relate the energy used by the primary network.

However, the applications of bioelectrical studies are variable, depending on the medical requirement, the analysis must be adapted. On the one hand, we have the circumstance where the study has a set of nodes that monitor a specific area. Consequently, the priority of some sensors is greater than the rest. In this way, the communication and analysis scheme focuses on the priority that each sensor has.

In the other hand, there is a primordial interest on the network energy optimization. In that case, it is much more convenient to modify the perspective so that the analysis is applied on the lifetime of the batteries and their respective residual energy.

\subsection{Priority Based Scheme Model}

There are scenarios where one sensor has more importance than the others. For example, in an EGG, there is an area with greater gastric movement and takes precedence over the rest. For this case, the first scheme based on priorities is proposed.

This scheme considers an amount of $M$ continuous monitoring nodes and $n_{e}$ event-monitoring. In each frame, on and off time will be generated. When some node in the primary network is off, then secondary network information can be transmitted through one of the four nodes that monitor the stomach. Each of these nodes will have an assigned priority. Only two priorities are presented: high or low. To differentiate them, each of them will have an assigned transmission probability, the one of high priority will be greater than the others. Therefore, it is proposed: Transmission probability of high priority node $=0.85$ and Transmission probability of low priority node $=0.25$.

Each node will have its own transmission probability determined by its respective priority, so $\rho_{i}$ represents the probability of transmission of an $i$-event monitoring node. Based on the above, there will be three possible cases:

1 . One high priority node and three low priority nodes $\left(\rho_{0}=0.85, \rho_{1}=0.25, \rho_{2}=\right.$ 0.25 and $\rho_{3}=0.25$ )

2 . Two high priority nodes and two low priority nodes $\left(\rho_{0}=0.85, \rho_{1}=0.85, \rho_{2}=\right.$ 0.25 and $\rho_{3}=0.25$ )

3 . Three high priority nodes and only one low priority node $\left(\rho_{0}=0.85, \rho_{1}=0.85\right.$, $\rho_{2}=0.85$ and $\rho_{3}=0.25$ )

It is necessary to indicate that the transmission probability of high or low priority is independent of the probability of successful transmission of the packet $(\epsilon)$. 


\subsection{Residual Energy Based Scheme Model}

Priority Data Transmission Schemes for a Wireless Sensor Network on BAN

When nodes that are monitoring are equally important, all nodes can transmit with the same priority. However, depending on the case, there are sensors that monitor much more than others, so the battery-lifetime of these becomes shorter. In other words, its efficiency and probability of sending the data will depend on how much energy is available from the battery. In this way the residual energy scheme is proposed. This second scheme shares the same basis as the priority scheme, it has a primary network of $M$ nodes and a secondary network composed of $n_{e}$ nodes. In this case, we will consider a transmission probability dependent of the energy still holding the battery for each sensors. This is called residual energy and is directly related to the probability of transmission of each of the event-monitoring node.

$$
\rho i=\gamma e-\gamma E 0 / E R,
$$

where $\rho_{i}=$ transmission probability for i-node, $\gamma=$ Battery decay factor, $E_{0}=$ Initial battery energy, $E_{R}=$ Residual battery energy

It is again emphasized that the probability of transmission by residual energy is independent of the probability of successful transmission ().

\section{WSN Performance Simulations And Results}

The network performance was applied by discrete event simulations. The results will be display for the primary and secondary network divided into the two proposed schemes (by priority and by residual energy). The scale for energy consumption depends on lifetime battery of $1 * 10^{9} E_{[t]]}$.

All the graphs shown below are determined by the variables: $P_{O N}$ which represents the on time probability per frame and which indicates the probability that an event is successfully transmitted.

\subsection{Priority Based Scheme Model}

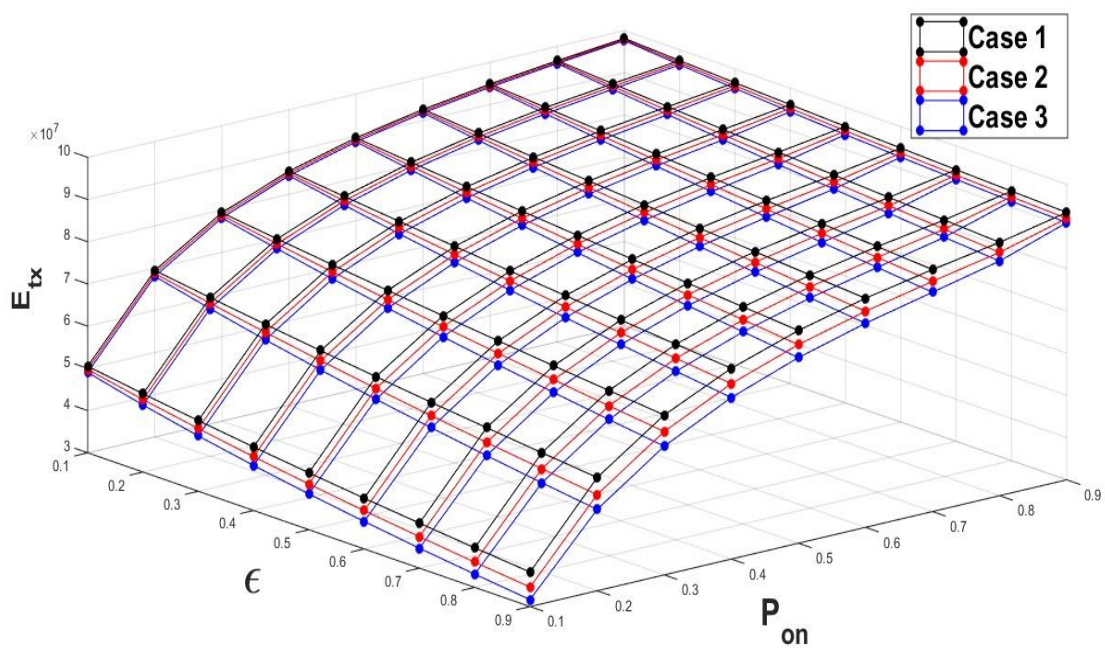

Fig.1. Primary Network Energy consumption (active nodes). 


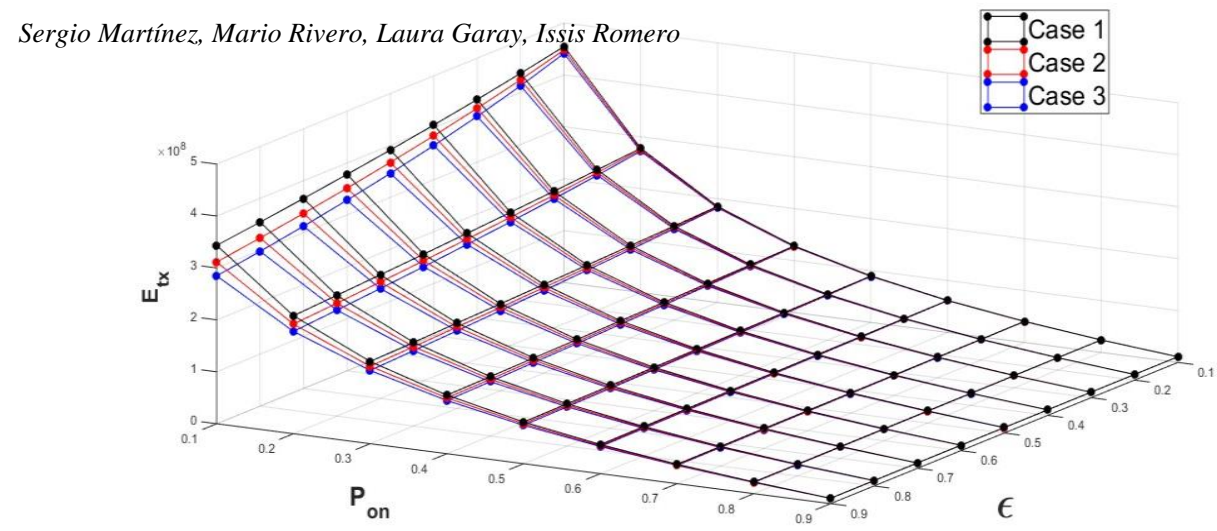

Fig.2. Primary Network Energy consumption (sleep mode nodes).

In these first figures it is possible to show the behavior of the primary network respect to the energy consumption for active and sleep nodes. These graphs show the three possible cases identified in section 3.2. Specifically, it can be considered that as the on time probability increases, the amount of energy consumption increases. It can also be denoted that for the minimum turned-on time and a maximum probability of successful packet transmission, the scheme that has three high priority nodes and one low one presents the least amount of energy consumed. It is important to remind that Case 1: $\rho_{0}=0.85, \rho_{1}=0.25, \rho_{2}=0.25$ and $\rho_{3}=0.25$, Case 2: $\rho_{0}=0.85, \rho_{1}=0.85, \rho_{2}=0.25$ and $\rho_{3}=0.25$ and Case 3: $\rho_{0}=0.85, \rho_{1}=0.85$, $\rho_{2}=0.85$ and $\rho_{3}=0.25$

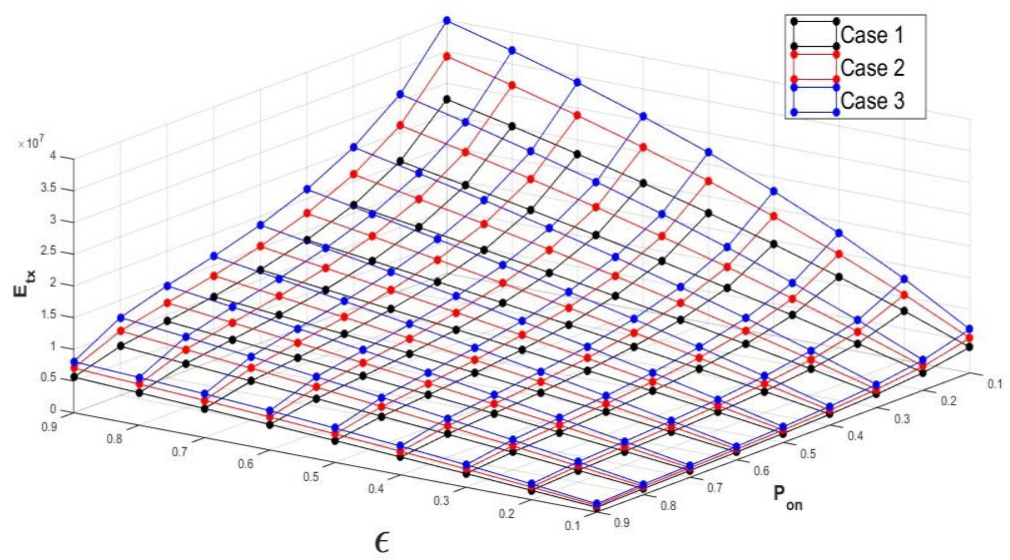

Fig.3. Secondary Network Total Energy Consumption.

For the secondary network it can be seen that the system has a maximum energy consumption when the on time is very low and the probability of successful transmission is high. Although within the three cases, the scheme of three high priority and one low node is the one that requires the maximum energy consumption, so this scheme is not optimum when there is a large amount of monitoring per event. 


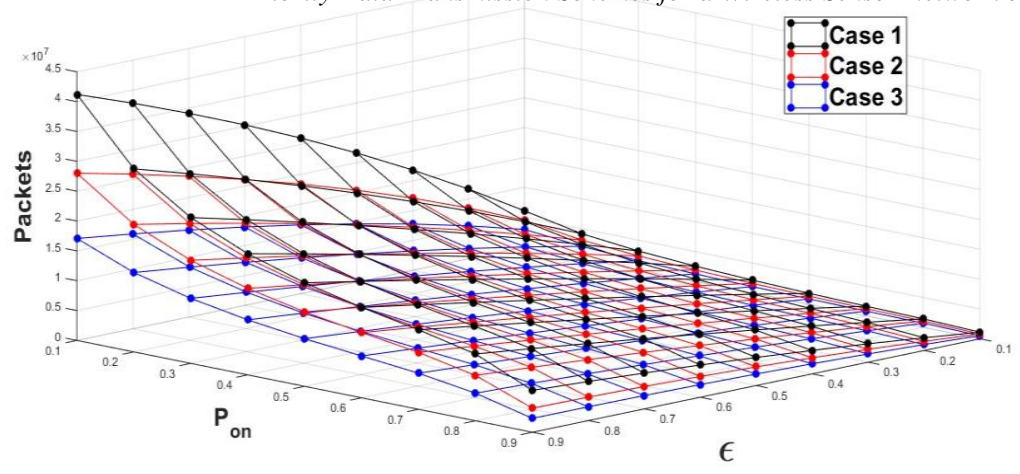

Fig.4. Network Packet Loss (non reported packets).

In the subsequent figure, it can be seen the loss of packets due to a lack of reporting (i.e., not falling into the probability of transmission by priority, they are not transmitted.) In this sense, it can be denoted that the maximum number of lost packets are when there is only one high priority sensor.

\subsection{Residual Energy Based Scheme}

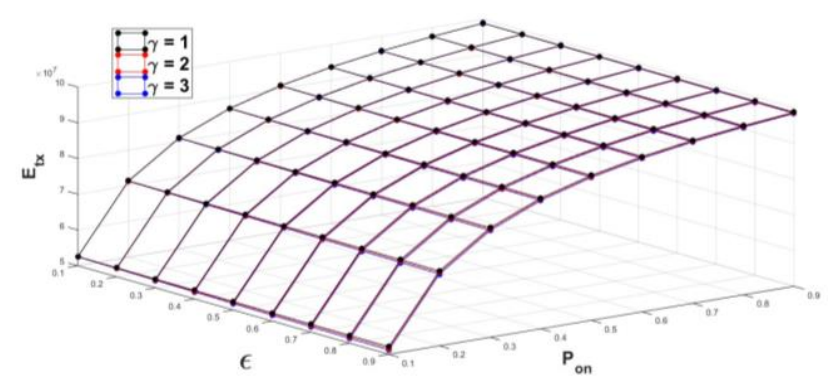

Fig.5. Primary Network Energy consumption (active nodes).

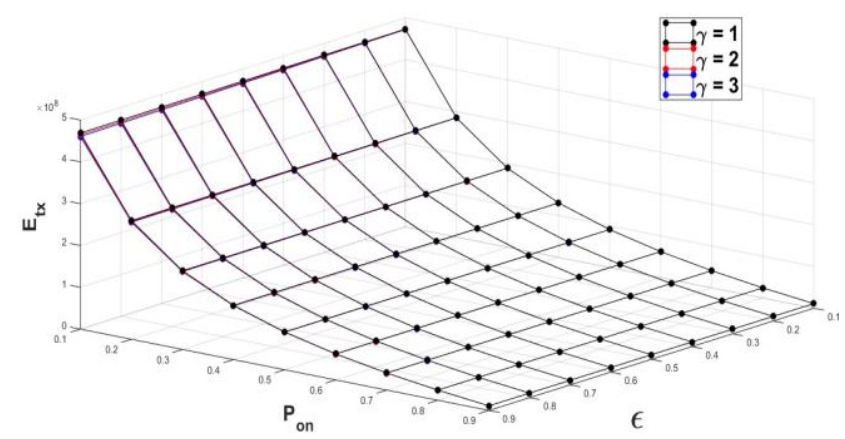


In these figures we present a behavior quite similar to the system by priorities,

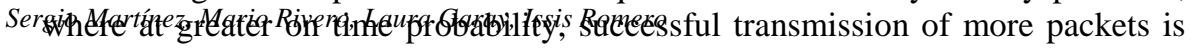
shown and the energy used in active nodes is greater. However, unlike the priority scheme, the energy consumption for this network is lower.

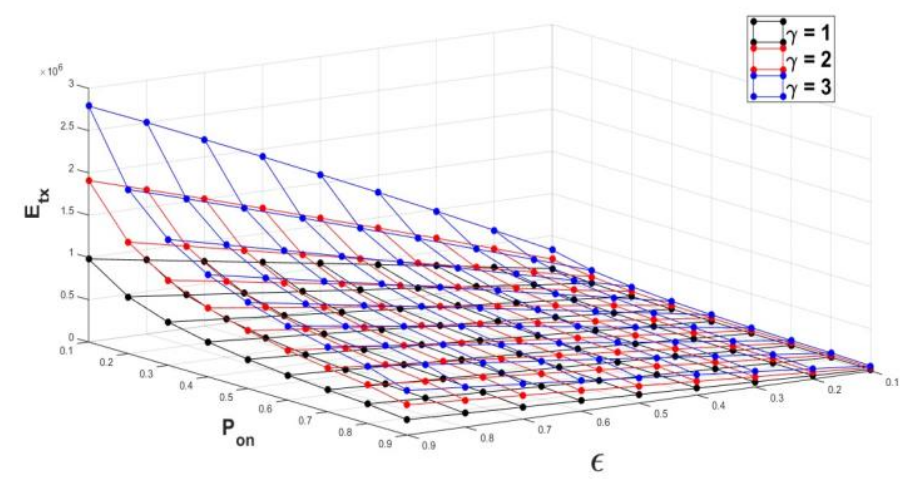

Fig.7. Secondary Network Total Energy Consumption.

The secondary network in this case shows a considerable difference with respect to the decay factor of the battery, where the higher the factor, the higher the energy consumption obtained by the secondary network.

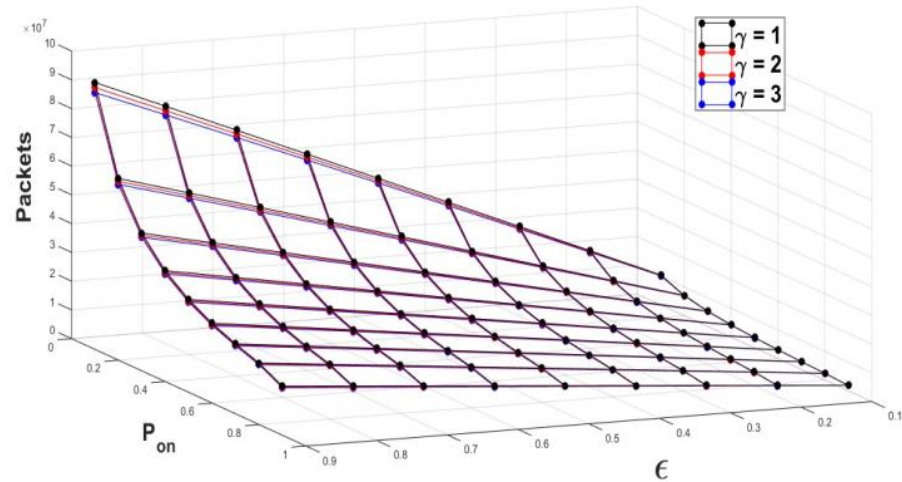

Fig.8. Network Packet Loss (non reported packets).

Regarding the loss of packets, it can be denoted that the behavior for the different battery decay factor is practically the same, where the greatest loss occurs when there is the maximum information transmission request and the minimum amount of on time probability. It should be considered that the packet loss here involves the packets of the primary network.

\section{Conclusions}

In the present research a WSN focused on biopotential studies was proposed that works with TDMA deterministic protocol and uses cognitive radio through op
Research in Computing Science 138 (2017) 
processes to reduce the number of slots used on each frame. Energy performance

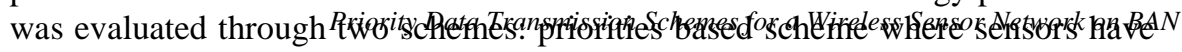
different importance (some body parts are more important than the others) and residual energy scheme where the aim is to decrease the battery energy consumption. The network is represented by discrete event simulations.

Therefore, a priority scheme can be considered to generate lower energy consumption in the primary network, more variable consumption in the secondary network but more losses than the residual energy scheme. If the application requires few monitoring per event, it is advisable to use a scheme based on priorities, otherwise the residual energy scheme is recommended.

\section{References}

1. Nakajima, T.: Aspects of Information Communications Technology for Better Medical Control, International Journal of E-Health and Medical Communications (IJEHMC). 1, 18-27, (2010)

2. Mahmoudi, R., Inievski, K.: Low power emerging wireless technologies, 2, CRCPress, (2012)

3. Dargie, W., Poellabauer, C.: Fundamentals of Wireless Sensor Network. Chichester: John Wiley \& sons Ltd, p. 34, (2010)

4. Castillejo, P., Martínez, J., Rodríguez, M.: Integration of wearable Devices in a wireless sensor network for an e-health application. Institute of Electrical and Electronics Engineers, 13, 38-49 (2013)

5. Shaikh, A., Pathan, S.: Research on Wireless Sensor Network Technology. International Journal of Information and Education Technology, 2, 476-479 (2012)

6. Chatzigiannakis, I., Kinalis, A., Nikoletseas. S.: Wireless sensor networks protocols for efficient collision avoidance in multi-path data propagation. In: Proceedings of the 1st ACM international workshop on Performance evaluation of wireless ad hoc, sensor, and ubiquitous networks (PE-WASUN '04), ACM, 8-16 (2004)

7. Sankarasubramaniam, Y., Akyildiz, I., McLaughlin, S.: Energy Efficiency Based Packet Size Optimization. In: Wireless Sensor Networks IEEE International Workshop on Sensor Network Protocols and Applications, 1-8 (2003)

8. Rodriguez, J.: Digital Advances in Medicine, E-health, and communication technologies. Digital: IGI Global, p. 12 (2013)

9. Dargie, W., Poellabauer, C.: Fundamentals of Wireless Sensor Network. JohnWiley \& sons Ltd: Chichester, p. 24 (2010)

10. Ganong, W.: Review of Medical Physiology. McGrawHill: New York, p. 576 (2005)

11. Enderle, J., Blanchard, S., Bronzino, J.: Introduction to biomedical engineering. Elsevier academic press: California, p. 323 (2004)

12. Martinez, S., Rivero, M., Garay, L.: Design of hybrid wireless sensor network to monitor bioelectric signals focused on the study of epilepsy. Research in Computing Science, 75, 43-49 (2014)

13. Martinez, S., Rivero, M., Garay, L.: Performance Analysis of Preemptive and NonPreemptive Schemes in Hybrid Wireless Sensor Networks focused on the study of epilepsy. Research in Computing Science, 101, 29-42 (2015) 